\title{
EFFICIENT CHARACTERIZATION AND MODELLING OF THE NONLINEAR BEHAVIOUR OF LFT FOR CRASH SIMULATIONS
}

\author{
HANNES GRIMM-STRELE ${ }^{1}$, MATTHIAS KABEL ${ }^{1}$, HEIKO ANDRÄ ${ }^{1}$, SARAH \\ STAUB $^{1}$, JÖRG LIENHARD ${ }^{2}$, TIMO SCHWEIGER ${ }^{2}$ AND OLAF HERD ${ }^{3}$
}

\author{
${ }^{1}$ Fraunhofer Institute for Industrial Mathematics (ITWM) \\ Fraunhofer-Platz 1, 67663 Kaiserslautern, Germany \\ hannes.grimm-strele@itwm.fraunhofer.de,matthias.kabel@itwm.fraunhofer.de, \\ heiko.andrae@itwm.fraunhofer.de, sarah.staub@itwm.fraunhofer.de,www.itwm.fraunhofer.de \\ ${ }^{2}$ Fraunhofer Institute for Mechanics of Materials (IWM) \\ Wöhlerstraße 11, 79108 Freiburg, Germany \\ joerg.lienhard@iwm.fraunhofer.de, timo.schweiger@itwm.fraunhofer.de, www.iwm.fraunhofer.de \\ ${ }^{3}$ Celanese Services Germany GmbH \\ Olaf.Herd@Celanese.com
}

Key words: computational mechanics, composites, LFT, microscale, viscoplasticity, viscoelasticity, damage.

Abstract. Modeling the nonlinear material behaviour of long fiber reinforced thermoplastics (LFT) presents a challenging task since local inhomogeneities and nonlinear effects must be taken into account also on the microscale. We present a computational method with which we can predict the nonlinear material response of a composite material using only standard DMA measurements on the pure polymer matrix material.

The material models considered include plasticity, damage, viscoelasticity, and viscoplasticity as described in [1]. These models can be combined similar to the model from [2] and extended to the composite by assigning linear elastic properties to the fibers. The mechanical response of the composite is computed using an FFT-based technique [3].

The geometry of the composite, in particular the fiber orientation, can be characterized using injection molding simulations or micro CT scans. We create virtual models of the composite using the algorithm of [4]. We show that with this method, the material behaviour of the composite can be predicted while the experimental complexity needed for the material characterization is low.

\section{INTRODUCTION}

Injection-molded long fiber reinforced thermoplastics (LFT) are often used in crash-relevant components due to their stiffness and strength properties. However, the prediction of the mechanical behaviour of these components is complicated due to the directional dependence.

The current state of the art is to capture the interaction between the microstructure and macroscopic behaviour using multiscale methods $[4,5]$. Multiscale methods allow the direct 
coupling of microscale simulations to the macroscale. FeelMath [3, 6-9] is a fast and efficient analysis tool for the calculation of effective mechanical and thermal properties on the microscale.

In multiscale methods, the averaged fiber orientation on the macroscale is modelled by representative volume elements (RVE) on the microscale. The Sequential Addition and Migration method (SAM) allows the generation of volume elements of LFT microstructures with prescribed fiber orientation tensor and high solid volume fraction [10].

In this work, we present the computational modelling of nonlinear (in particular strain rate dependent) material behaviour of an injection-molded long fiber reinforced plate with minimal experimental effort. With FeelMath, we can simulate the mechanical behaviour of the composite on RVEs created using the geometrical characterization obtained from the CT scans. In the segmented CT scan, fibers and matrix material are separated. We assign an isotropic linear-elastic material law to the glass fibers. For the matrix material, we use an isotropic nonlinear material law. As described in the following sections, the material parameters are calibrated using DMTA measurements on the matrix material. The type of calibration experiment needed depends on the material law. The parameters are optimized by an automatic procedure based on the Nelder-Mead algorithm to reproduce the DMTA measurements. The material behaviour of the composite is validated using the tensile tests of the composite. The anisotropy of the behaviour of the composite is captured by the mean fiber orientation of the microscale models.

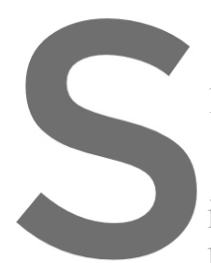

1.1 Geometrical Characterization
The composite material we investigate is Sabic STAMAX
is Polypropylene (PP) to which 30 weight- $\%$ glass fibers are acd
length is $1.3 \mathrm{~mm}$, while the volume averaged flber length is 2 .

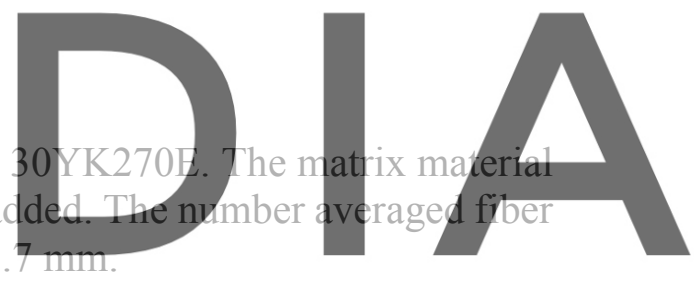

We mold a plate with $2 \mathrm{~mm}$ thickness with this material and extract three samples at the

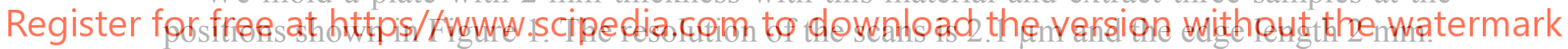
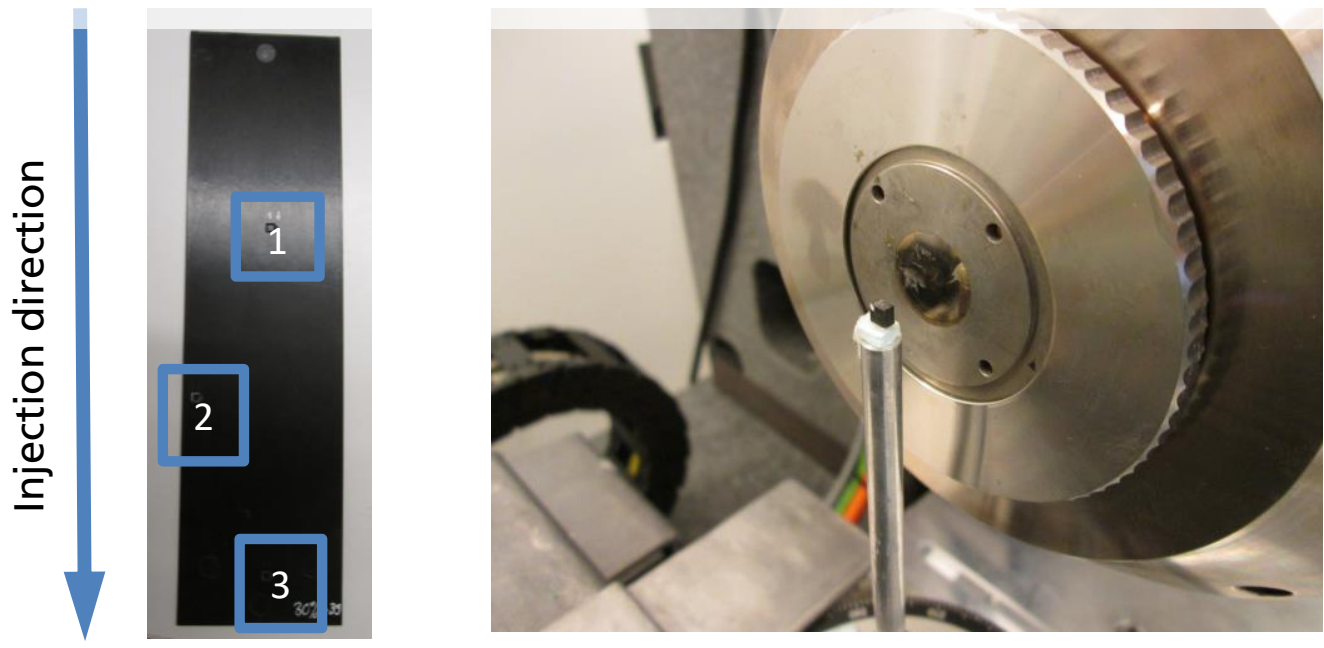

Figure 1: left: plate geometry and sample location; right: CT scanner. 
The fiber orientation in the scans is analyzed in GeoDict [11]. In the CT scans, we observe that near the center, a middle layer is formed where the fiber orientation is inverted. As shown in Figure 2, the middle layer is stronger in sample 3 than in sample 1. In sample 2 (near the border of the plate), the fiber orientation is nearly uniform.
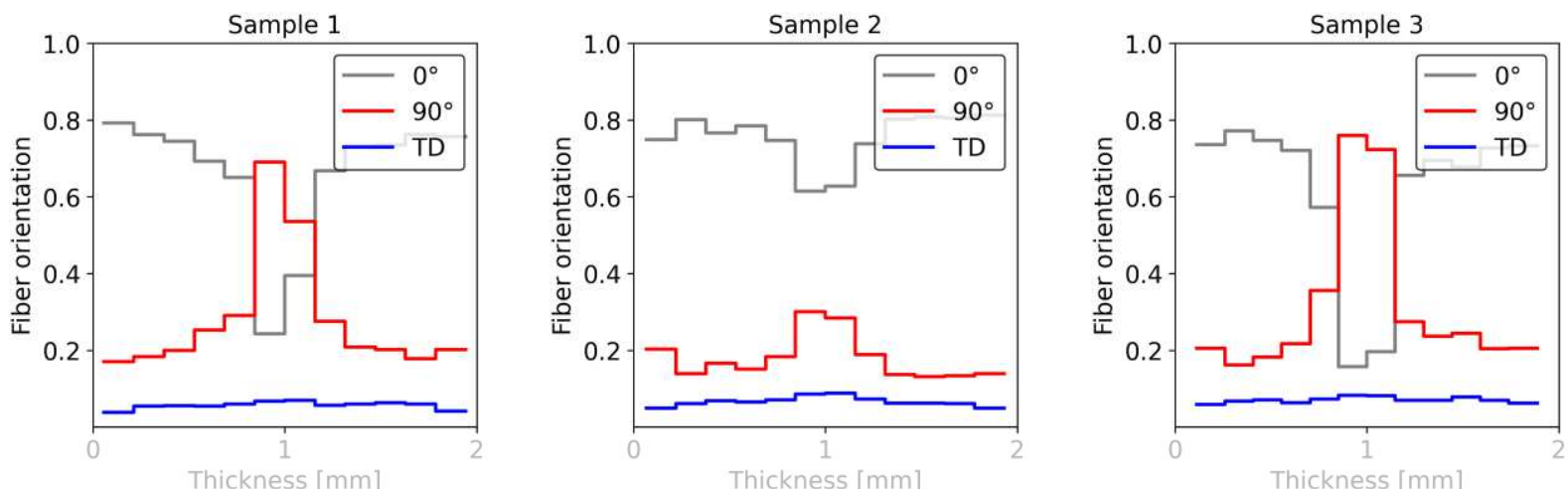

Figure 2: Fiber orientations calculated from the scans of the three samples from the plate. "00" refers to the injection direction, " $20^{\circ}$ " to the perpendicular direction, "TD" to the thickness direction.

\subsection{Experimental Characterization}

The material behaviour is experimentally characterized by several tensile tests, which serve as validation data for our method. The results are shown in Figure 3. We observe that the material behaviour is

- Direction dependent: the

- Strain rate dependent: dyr

- Location dependent: variations between n
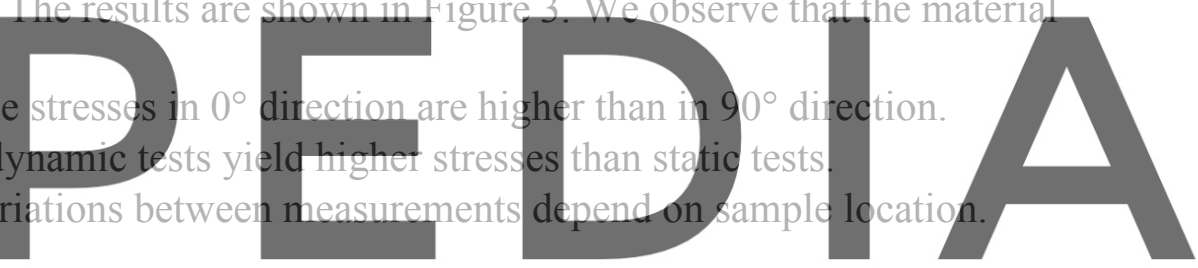

Register for free at https//wwW.scipedia.com to dowinlbad the version without the watermark
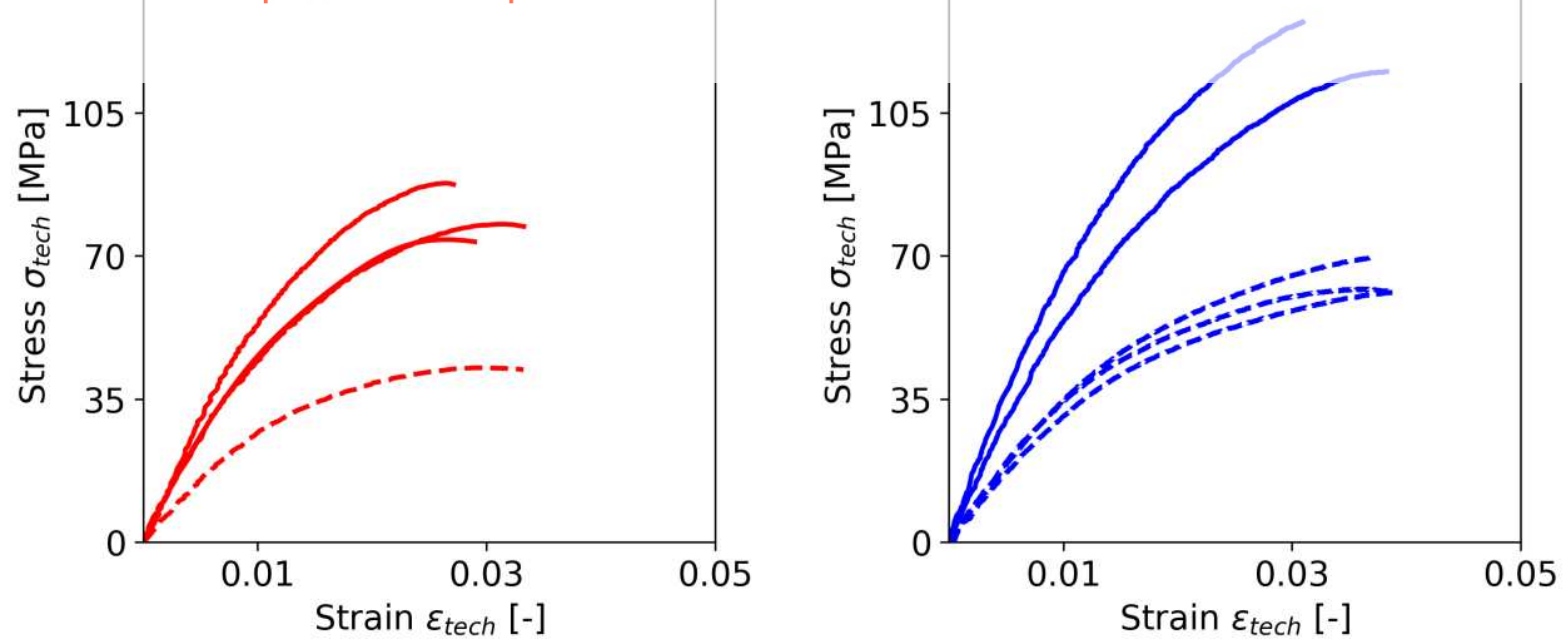

Figure 3: Strain-stress curves obtained from experiments. Solid lines: $0^{\circ}$ orientation, dashed lines: $90^{\circ}$ orientation; static strain rate: $\sim 10^{-3} \mathrm{~s}^{-1}$, dynamic strain rate: $\sim 10^{1} \mathrm{~s}^{-1}$. 


\section{PARAMETER CALIBRATION}

In order to model the nonlinear material behaviour of the composite, we start by calibrating parameters for the matrix material. Tensile measurements were carried out at the ITWM on 10 mm wide test specimens on the DMTA (dynamic mechanical thermal analyzer). The Gabo Netzsch Eplexor 500N is available at the ITWM for this purpose. With the aid of the DMTA, decoupled static loads (up to $1500 \mathrm{~N}$ ) and additional cyclic loads (up to $500 \mathrm{~N}$ ) can be applied at specified frequencies up to $100 \mathrm{~Hz}$. The temperature can be adjusted in a range between -150 ${ }^{\circ} \mathrm{C}$ and $500{ }^{\circ} \mathrm{C}$.

To calibrate the material parameters for this project, cyclic loading and unloading tests were carried out for three strain rates, $0.05 \% / \mathrm{min}, 0.1 \% / \mathrm{min}$ and $1 \% / \mathrm{min}$. For this purpose, a force is applied to the tensile specimen for the respective strain rate and then unloaded again. The applied load is increased in the next cycle. From the stress-strain curves obtained in this way, the proportion of plastic strain per cycle can be identified by the permanent strain in the unloaded state. The damage developed per load cycle is also obtained from the stress-strain diagram and can be obtained by reducing the slope of the curve per cycle [12]. The plasticity and damage parameters determined by means of a Nelder-Mead optimization are summarized in Table 1.

Table 1: Calibrated material parameters for isotropic hardening and damage.
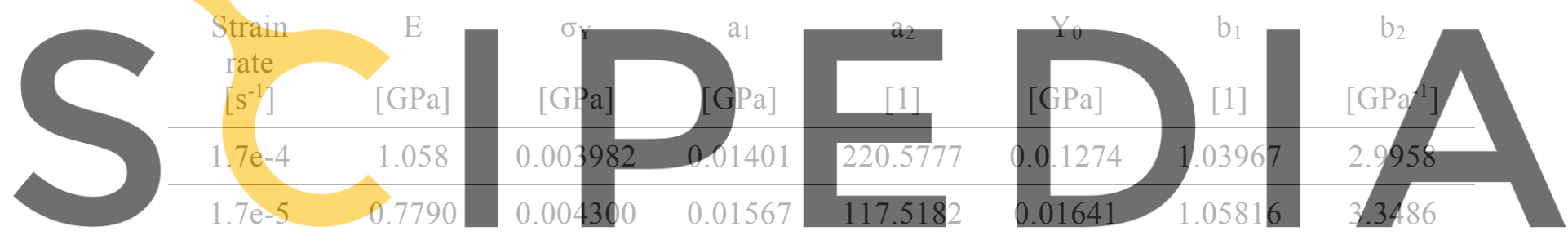

$8 \mathrm{e}-6$

0.6545

0.004736

0.01020

130.3810

0.01723

1.07264

3.9305

Register for free at https//www.scipedia.com to download the version without the watermark
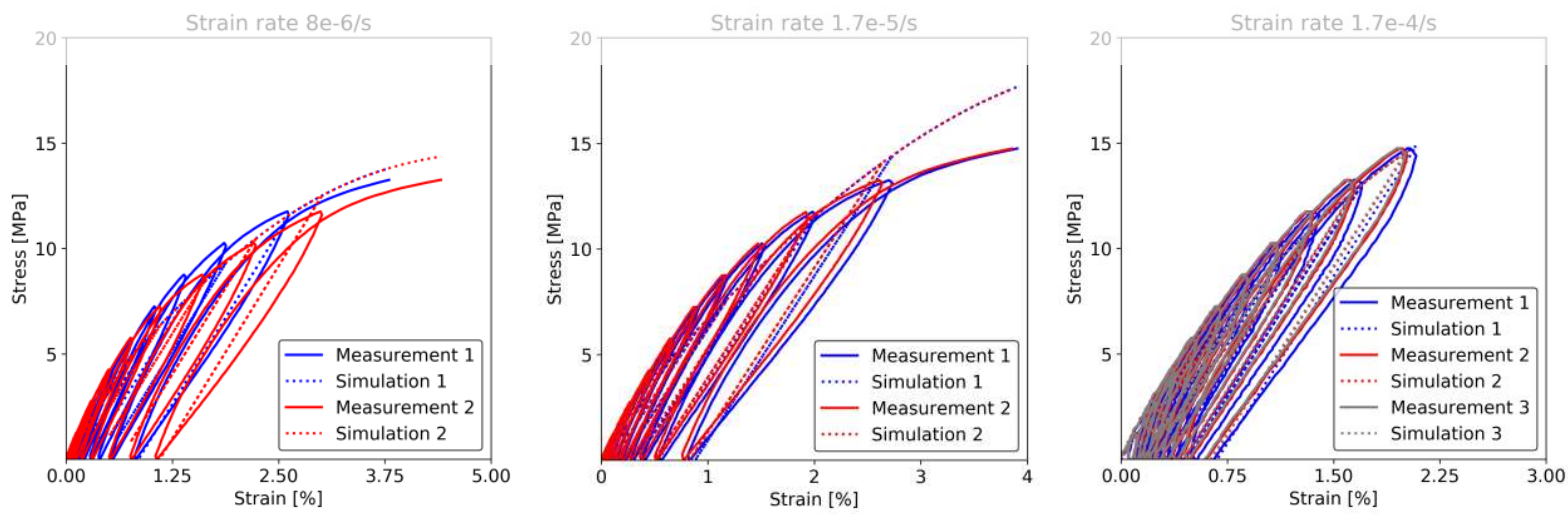

Figure 4: Loading-unloading tests and simulation results with fitted material parameters. Left: Strain rate $0.05 \% / m i n$. Middle: Strain rate $0.1 \% / \mathrm{min}$. Right: strain rate $1 \% / \mathrm{min}$.

The parameters for a viscoplastic model, here the Michel-Suquet model [13], can be calibrated using the same optimization algorithm based on tensile measurements with different strain rates. The parameters are shown in Table 2 . 
Table 2: Calibrated material parameters for the Michel-Suquet model.

\begin{tabular}{llll} 
Parameter & $\dot{\varepsilon}\left[\mathrm{s}^{-1}\right]$ & $\mathrm{n}[1]$ & $\sigma_{0}[\mathrm{GPa}]$ \\
\hline Value & 1.0 & 5.8349 & 0.0096818
\end{tabular}

To calibrate the viscoelastic properties, so-called master curves based on temperaturefrequency sweeps were generated in addition to the loading and unloading tests. For this purpose, 25 frequencies between $0.5 \mathrm{~Hz}$ and $50 \mathrm{~Hz}$ were applied in the tensile test at temperatures between $10{ }^{\circ} \mathrm{C}$ and $130{ }^{\circ} \mathrm{C}$, and the dynamic moduli (storage and loss modulus) were determined in the linear elastic range. Using the time-temperature superposition principle, low frequency ranges can be estimated from measurements of higher temperatures and, conversely, high frequency ranges can be estimated from measurements at lower temperatures. This allows the master curve to be generated for a wide range of frequencies at a chosen reference temperature $\left(20^{\circ} \mathrm{C}\right.$ in this case).

In the viscoelastic model developed in [14], several Maxwell elements are connected in parallel to form a generalized Maxwell element. The parameters calibrated using the measurements described above are summarized in Table 3.

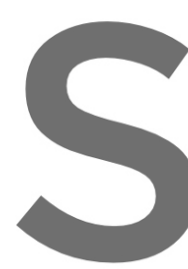

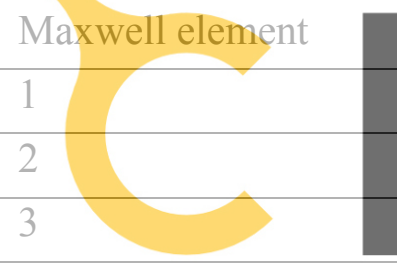

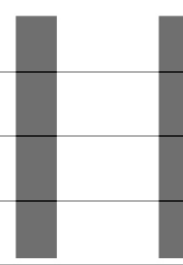

4

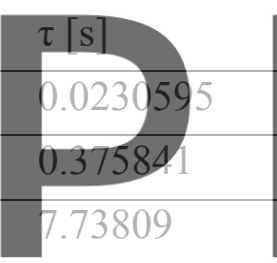

142.133

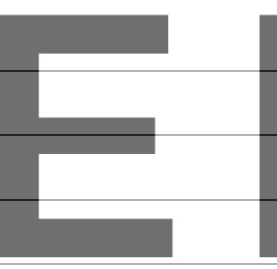

0.0946933

Register for free at https//www.scipedia.com to download the version without the watermark

Similar to [2], this model can be extended to include plasticity, damage, and viscous elements.

In this project, the elastic Hooke element was replaced by a corresponding viscoplastic element with damage.

In the simulation, these effects can be combined as desired. To illustrate their effects, tensile tests with different strain rates on a homogeneous structure with pure PP were calculated with FeelMath. For this purpose, three tensile tests on the Sabic material with low strain rates and three tensile tests on a similar material from Celanese with high strain rates were combined. In Figure 5, measurement results are always shown with solid lines and simulation results with dashed lines. 


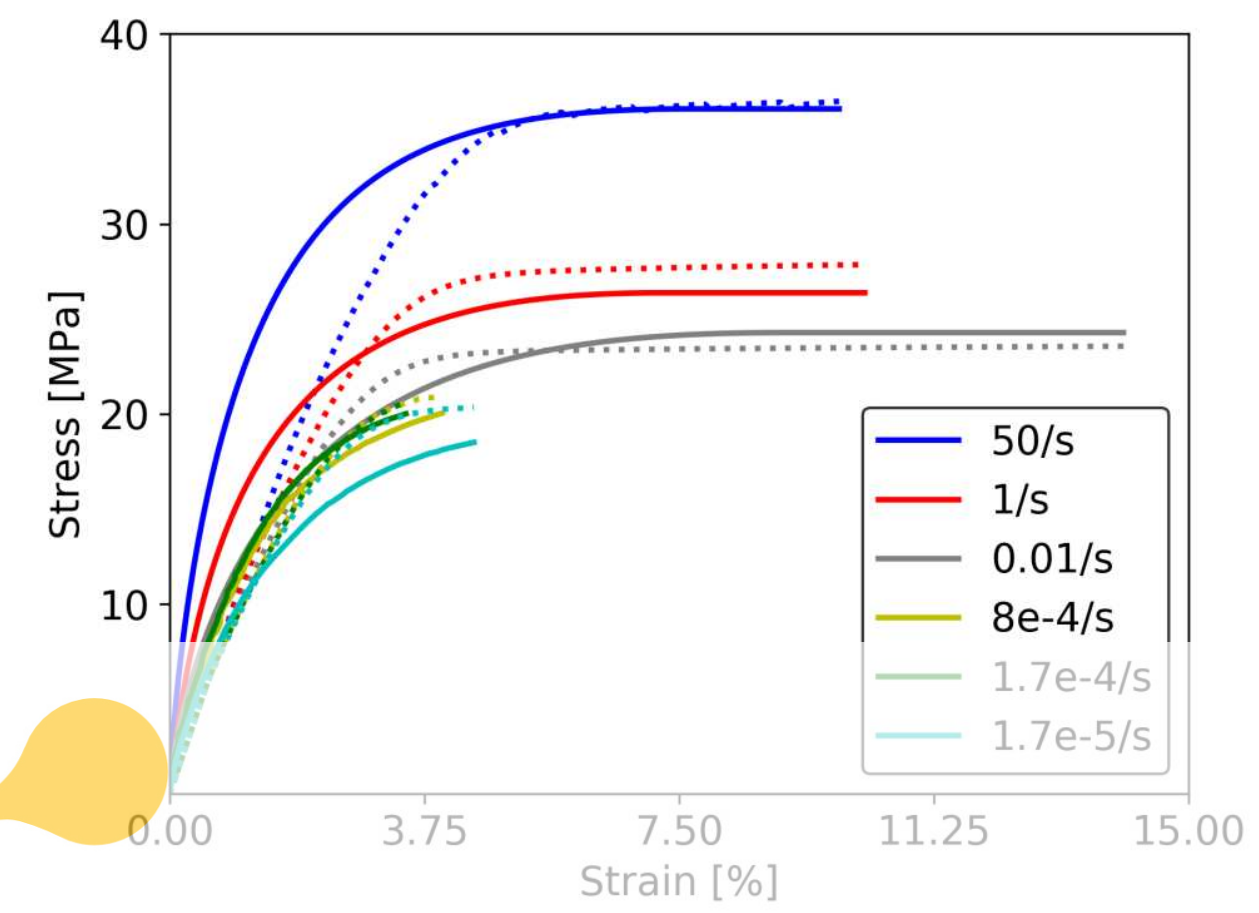

Figure 5: Stress-strain curves with viscoplasticity (Michel-Suquet model), damage and viscoelasticity.

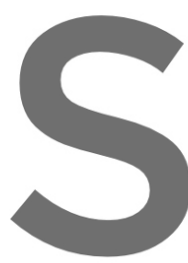

\section{The combined viscoe} of the measured materi strain-rate dependent. stiffness. Due to the This model can best rep
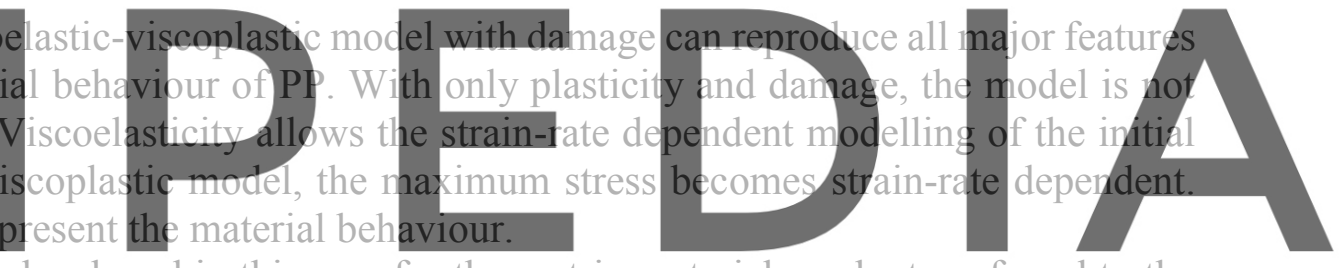

The material model developed in this way for the matrix material can be transferred to the

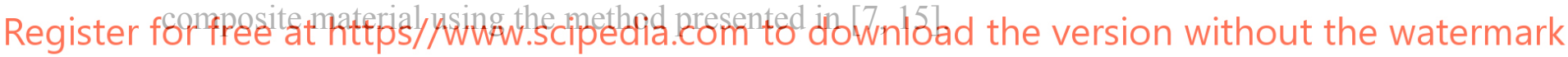

\section{MICROSTRUCTURE SIMULATIONS}

Using the parameters determined in the previous section, microstructure simulations can be performed directly on the segmented $\mu \mathrm{CT}$ scans. For this purpose, linear elastic behaviour $(\mathrm{E}=$ $72 \mathrm{GPa}, v=0.22$ ) is assumed for the fibers, while isotropic hardening with damage was set as the material model for the matrix. 

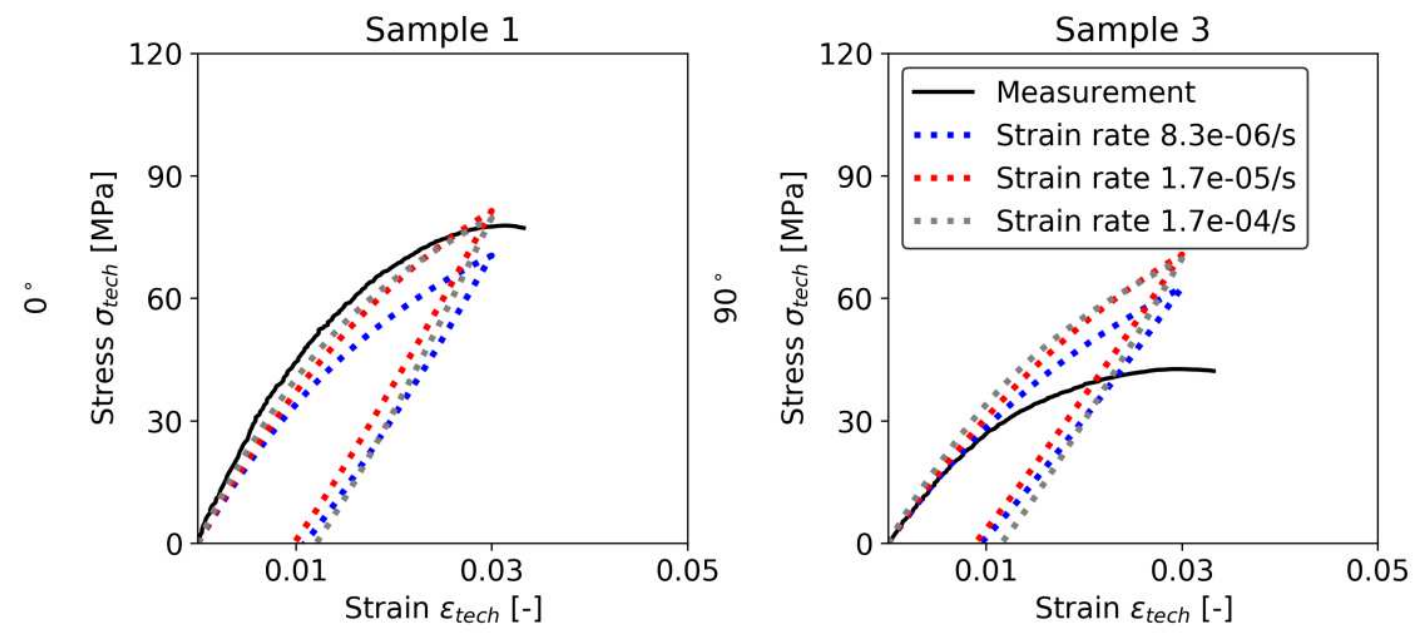

Figure 6: Stress-strain curves with isotropic hardening and damage on the plate samples from the composite material.

Results with the parameter sets of PP from Table 1 are shown in Figure 6. While for $0^{\circ}$ direction, the simulation results show good agreement with the measurements, the results are clearly too stiff in the $90^{\circ}$ direction. The parameters determined from the $1 \% / \mathrm{min}$ tests give the closest results to the measurement performed at $6 \% / \mathrm{min}$ strain rate and will be our default choice from now on.

Despite the high resolution of the $\mu \mathrm{CT}$ scans, the fibers cannot always be completely separated, especially when the fiber volume content is high. Figure 7 shows on the right side in blue an example of a segmented image. These fibers produce an u simulation results. Since the denter layer has effect is strongest in the be separated in the segmented $\mu \mathrm{CT}$ image.

Register for free at https//www.scipedia.com to download the version without the watermark
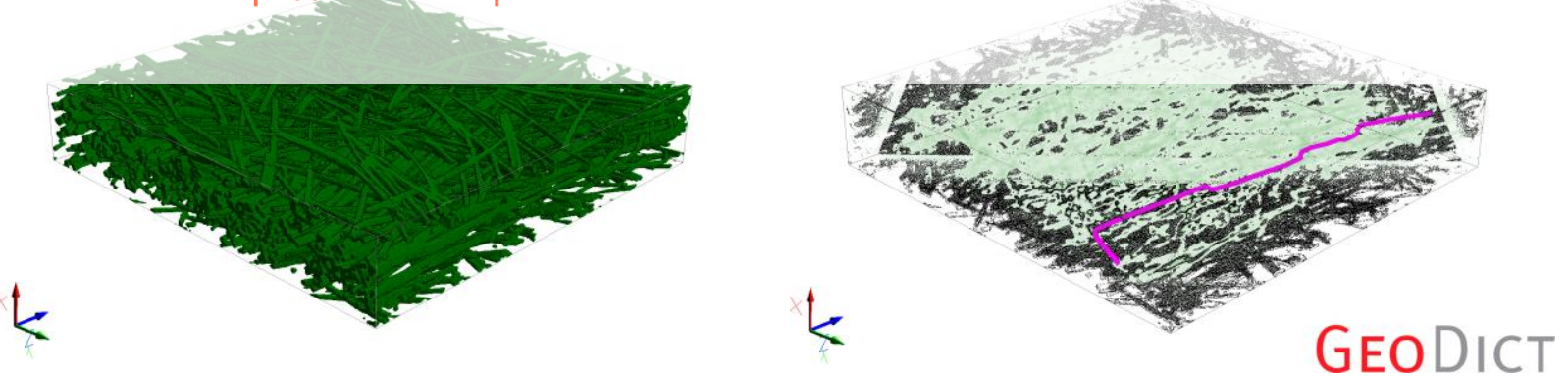

Figure 7: Left: $\mu$ CT image of the middle layer of sample 3. Right: example of a fiber detected as coherent (in blue).

\subsection{Generation of "digital twins"}

Using the data from the CT analysis, virtually generated microstructure models, so-called "digital twins", will be generated and the nonlinear material behaviour of the composite material will be modeled. In the digital twin, all fibers are separated. The result is a parameterized simulation model for the composite material.

In most CT scans, a middle layer is visible, where the fibers are located at $90^{\circ}$ to the injection 
molding direction and where the fiber volume content is higher (see Figure 2). Therefore, the virtual structure generator described in [10] was modified so that layered models, which have a different fiber orientation tensor and fiber volume content in each layer, can be generated. The input is the fiber orientations determined from the CT scans, which can be determined insensitive to the threshold value in the conversion from gray to binary values. The average fiber volume content can be set according to the manufacturer's specification.

Reliable values for fiber diameter and fiber length were determined by ashing of the composite. The distribution of fiber lengths is asymmetric and has a high variance. The number averaged fiber length was found to be $1.308 \mathrm{~mm}$ and the volume averaged fiber length 2.728 $\mathrm{mm}$.

To determine the correct fiber length distribution for the geometric models, we perform two parameter studies. We generate geometric models with the parameters listed in Table 4 . We choose as fiber length:

- Test series 1: a fiber length distribution with three fiber lengths and constant volume average (1 mm).

- Test series 2: variation of the average fiber length.

The fiber lengths used for series 1 are recorded in Table 5. We used isotropic hardening with damage as the material model for the matrix.
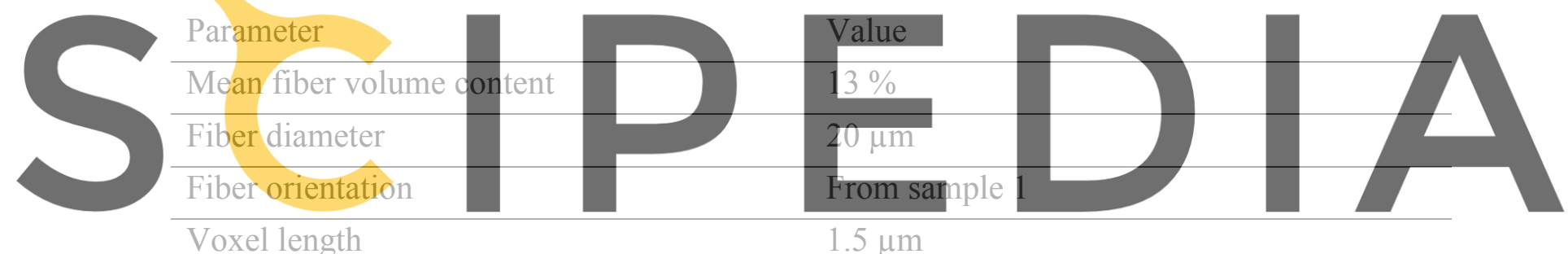

$1.5 \mu \mathrm{m}$

Register fabfreseat https//www.scipedia.com to doynmhad thenversiph without the watermark

Thickness of the middle layer

$0.192 \mathrm{~mm}$

Table 5: Fiber lengths of the geometric models used to study the influence of fiber length distribution.

\begin{tabular}{llll} 
& Fiber length 1 & Fiber length 2 & Fiber length 3 \\
\hline$\sigma=0 \mathrm{~mm}$ & $1 \mathrm{~mm}$ & - & - \\
\hline$\sigma=0.15 \mathrm{~mm}$ & $0.843 \mathrm{~mm}$ & $0.990 \mathrm{~mm}$ & $1.170 \mathrm{~mm}$ \\
\hline$\sigma=0.3 \mathrm{~mm}$ & $0.703 \mathrm{~mm}$ & $0.958 \mathrm{~mm}$ & $1.336 \mathrm{~mm}$ \\
& & & \\
\hline$\sigma=0.45 \mathrm{~mm}$ & $0.585 \mathrm{~mm}$ & $0.915 \mathrm{~mm}$ & $1.497 \mathrm{~mm}$
\end{tabular}

In Figure 8, we observe that a fiber length distribution has neither in the linear-elastic nor in the nonlinear regime any noticeable effects on the material response. Thus, only the volume average of the fibers is important in the geometric models. We can therefore assume a constant fiber length for the geometric models. 

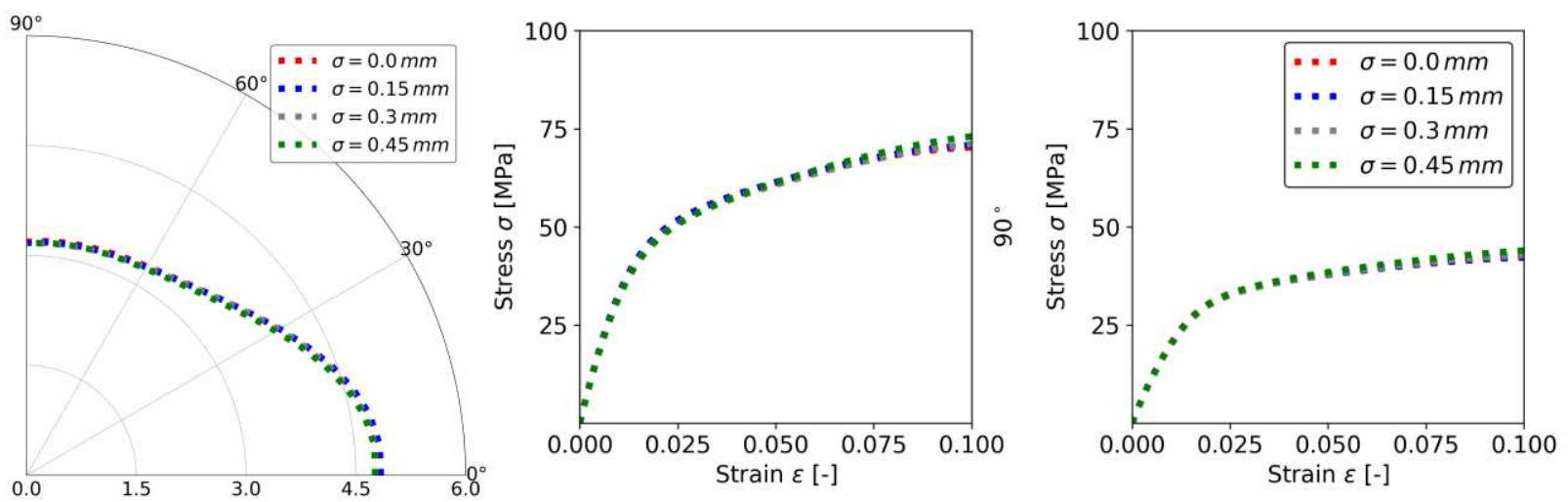

Figure 8: Material response for test series 1. Left: linear-elastic. Right: nonlinear.

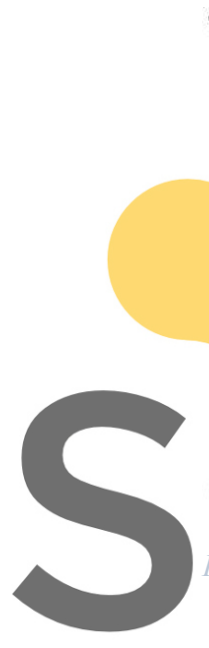

$90^{\circ}$
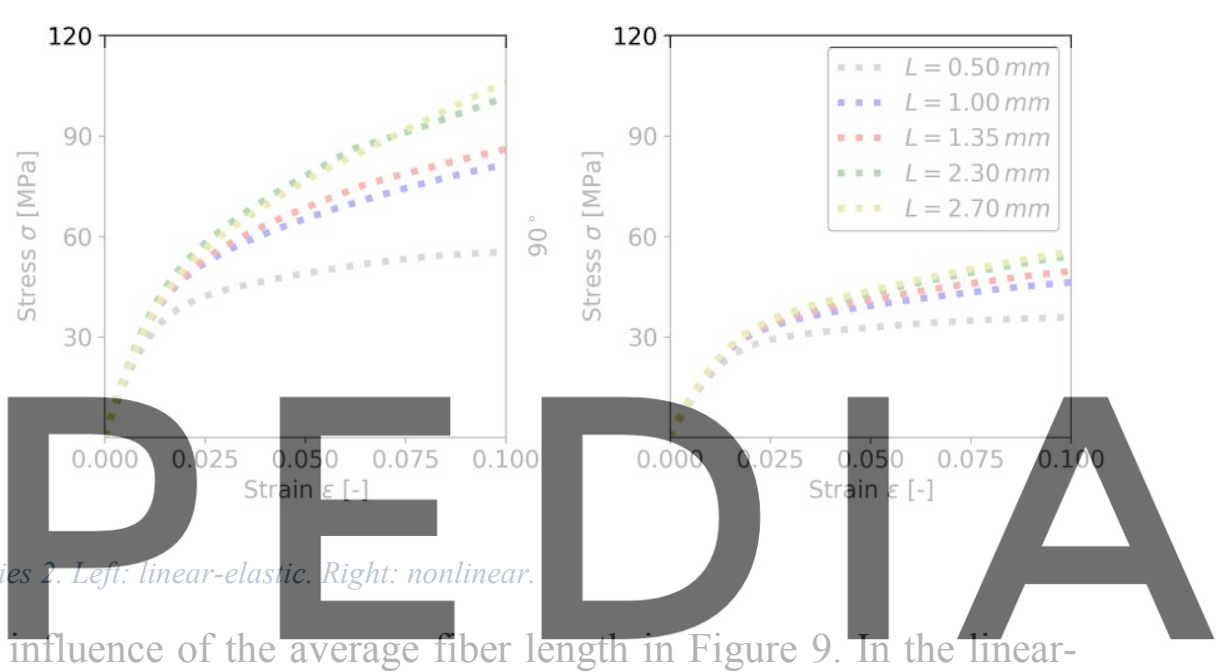

In contrast, we see a clear influence of the average fiber length in Figure 9. In the linear-

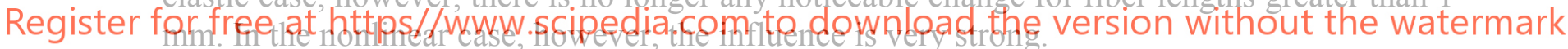

From this, we conclude that in the geometric models we can assume that all fibers are of equal length, and that the volume average determined by ashing shouid be used as the fiber length. Thus, the fiber length should be $2.7 \mathrm{~mm}$.

Lastly, the dependence of the geometric models on resolution, realization and the area size was investigated. The following guidelines for the geometric models can be derived:

- The resolution should be about 15 voxels per fiber diameter.

- The area size should be larger than 2 times the fiber length.

- Under these conditions, the influence of the realization (random process in the generation of the structure) is small.

To reduce the computational time of these simulations from weeks to days while obtaining accurate results, we use the composite voxel technique extended to nonlinear material laws [3].

\subsection{Validation on plate samples}

For samples 1 and 3 taken from the center of the plate, models with three layers were generated, while the model for sample 2, taken from the edge of the plate, has only one layer. On these models, the linear-elastic and the nonlinear behaviour of the fiber composite can be 
modeled and validated using the measurements from the previous section.

The stress-strain curves we obtain for these models agree well with the measured values, see Figure 10. The strain rate dependence as well as the maximum stress can be reproduced well with the viscoplastic or with the viscoelastic-viscoplastic model. In particular, when compared with the simulations on the $\mu \mathrm{CT}$ scans presented in Figure 6 , the stress-strain curves calculated on the geometric models are much closer to the measurements. The viscoelastic-viscoplastic model provides the best agreement with the measurements.

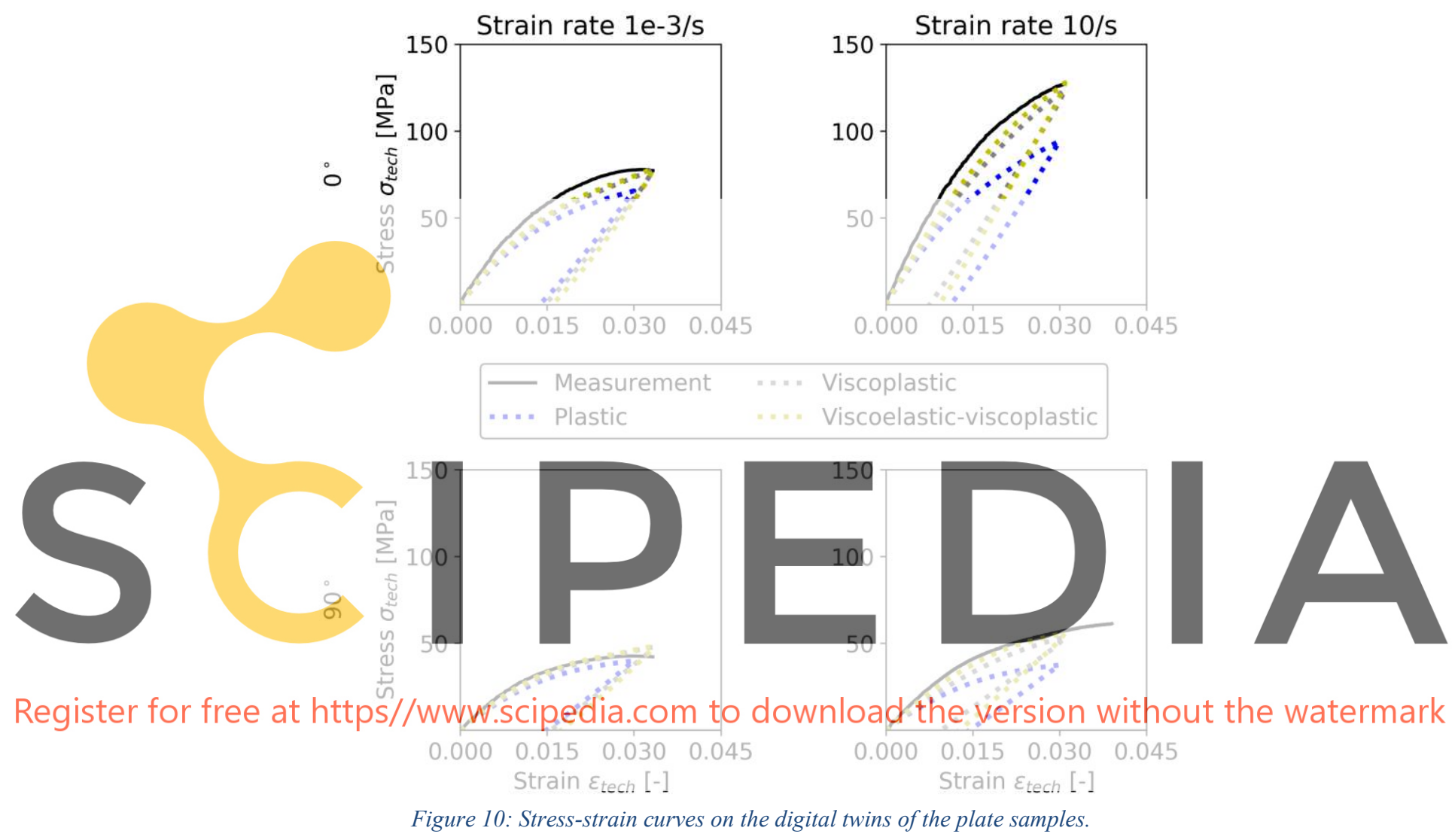

The comparison of the bottom-left panel in Figure 10 with Figure 6 clearly shows that the stresses in the simulation on the geometric model are significantly lower, since all fibers are perfectly separated here. This results in higher local matrix damage when calculating on the virtual model.

\section{CONCLUSIONS}

In this paper, we have presented an efficient and accurate way to characterize the geometry and material behaviour of a composite material. The main conclusions concerning the geometrical characterization are:

- The digital twin reflects the main geometrical features (e.g., fiber orientation) of the composite as identified in the CT scans.

- In the digital twin, all fibers are separated, whereas fibers which are erroneously sticked together lead to wrong simulation results. 
- Instead ofCT scans, injection molding simulations could provide the necessary input.

Concerning the physical characterization, we conclude:

- Several material models for the matrix (plastic, damage, viscoelastic, viscoplastic) can be calibrated using simple DMTA experiments.

- E.g., calibration of a viscoplastic law with damage only requires cyclic tests at a slow strain rate, and tensile tests at several strain rates.

- With our software tool FeelMath and the resulting material models, we can accurately reproduce the mechanical behaviour of the composite.

Once calibrated, the microscale model can be used for a variety of purposes. We can investigate of the influence of geometrical and physical parameters (e.g., fiber orientation, fiber length, yield stress). We can calibrate macroscopic material models by virtual experiments. Using multiscale methods, we can run macroscopic simulations on the component scale. Details can be found in [16].

\section{ACKNOWLEDGEMENTS}

We would like to thank Franz Schreiber for the computer tomography. The IGF project $19532 \mathrm{~N}$ of the Forschungsvereinigung Automobiltechnik e.V. (FAT) was funded by the German Federal Ministry for Economic Affairs and Energy (BMWi) via the AiF as part of the program for the promotion of joint industrial research and development (IGF) on the basis of a resolution of the German Bundestag.

\section{REFERENCES}

[1] G. Alfano, F. de Angelis, and L. Rosati, "General solution procedures in elasto/viscoplasticity," Computer Methods in Applied Mechanics and Engineering, vol. 190, no. 39, pp. 5123-5147, 2001, doi: 10.1016/S0045-7825(00)00370-4.

[2] P. Zerbe, B. Schneider, E. Moosbrugger, and M. Kaliske, "A viscoelastic-viscoplasticdamage model for creep and recovery of a semicrystalline thermoplastic," International Journal of Solids and Structures, 110-111, pp. 340-350, 2017, doi: 10.1016/j.ijsolstr.2016.10.029.

[3] M. Kabel, A. Fink, and M. Schneider, "The composite voxel technique for inelastic problems," Computer Methods in Applied Mechanics and Engineering, vol. 322, pp. 396-418, 2017, doi: 10.1016/j.cma.2017.04.025.

[4] J. Köbler, M. Schneider, F. Ospald, H. Andrä, and R. Müller, "Fiber orientation interpolation for the multiscale analysis of short fiber reinforced composite parts," Comput Mech, vol. 61, no. 6, pp. 729-750, 2018, doi: 10.1007/s00466-017-1478-0.

[5] J. Spahn, H. Andrä, M. Kabel, and R. Müller, "A multiscale approach for modeling progressive damage of composite materials using fast Fourier transforms," Computer Methods in Applied Mechanics and Engineering, vol. 268, pp. 871-883, 2014, doi: 10.1016/j.cma.2013.10.017. 
[6] H. Grimm-Strele and M. Kabel, "Runtime optimization of a memory efficient CG solver for FFT-based homogenization: implementation details and scaling results for linear elasticity," Comput Mech, vol. 64, no. 5, pp. 1339-1345, 2019, doi: 10.1007/s00466-01901713-3.

[7] M. Kabel, S. Fliegener, and M. Schneider, "Mixed boundary conditions for FFT-based homogenization at finite strains," Comput Mech, vol. 57, no. 2, pp. 193-210, 2016, doi: 10.1007/s00466-015-1227-1.

[8] M. Kabel, D. Merkert, and M. Schneider, "Use of composite voxels in FFT-based homogenization," Computer Methods in Applied Mechanics and Engineering, vol. 294, pp. 168-188, 2015, doi: 10.1016/j.cma.2015.06.003.

[9] M. Schneider, F. Ospald, and M. Kabel, "Computational homogenization of elasticity on a staggered grid,” Int. J. Numer. Meth. Engng, vol. 105, no. 9, pp. 693-720, 2016, doi: 10.1002/nme.5008.

[10] M. Schneider, "The sequential addition and migration method to generate representative volume elements for the homogenization of short fiber reinforced plastics," Comput Mech, vol. 59, no. 2, pp. 247-263, 2017, doi: 10.1007/s00466-016-1350-7.

[11] GeoDict, Math2Market GmbH, http://www.geodict.de, Kaiserslautern, Germany. Accessed: 2021-02-10.

[12] H. Andrä, D. Dobrovolskij, K. Schladitz, S. Staub, and R. Müller, "Modelling of Geometrical Microstructures and Mechanical Behaviour of Constituents," in Multi-scale Simulation of Composite Materials, S. Diebels and S. Rjasanow, Eds., Berlin, Heidelberg: Springer Berlin Heidelberg, 2019.

[13] J.-C. Michel and P. Suquet, "A model-reduction approach in micromechanics of materials preserving the variational structure of constitutive relations," Journal of the Mechanics and Physics of Solids, vol. 90, pp. 254-285, 2016, doi: 10.1016/j.jmps.2016.02.005.

[14] M. Kaliske and H. Rothert, "Formulation and implementation of three-dimensional viscoelasticity at small and finite strains," Computational Mechanics, vol. 19, no. 3, pp. 228-239, 1997, doi: 10.1007/s004660050171.

[15] S. Fliegener, T. Kennerknecht, and M. Kabel, "Investigations into the damage mechanisms of glass fiber reinforced polypropylene based on micro specimens and precise models of their microstructure," Composites Part B: Engineering, vol. 112, pp. 327-343, 2017, doi: 10.1016/j.compositesb.2016.12.051.

[16] H. Andrä et al., "Effiziente Charakterisierung und Modellierung des anisotropen Versagensverhaltens von LFT für Crashsimulation," FAT-Schriftenreihe 331, FAT, 2020, https://www.vda.de/de/services/Publikationen/fat-schriftenreihe-331.html. 